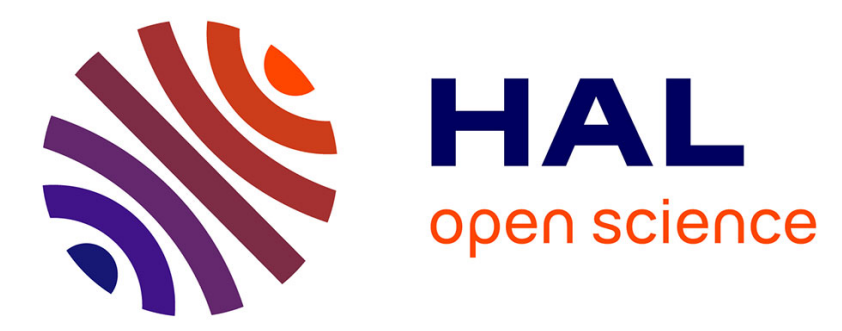

\title{
BIM-FM and Information Requirements Management: Missing Links in the AEC and FM Interface
}

\author{
Julie Jupp, Ramsey Awad
}

\section{To cite this version:}

Julie Jupp, Ramsey Awad. BIM-FM and Information Requirements Management: Missing Links in the AEC and FM Interface. 14th IFIP International Conference on Product Lifecycle Management (PLM), Jul 2017, Seville, Spain. pp.311-323, 10.1007/978-3-319-72905-3_28 . hal-01764191

\section{HAL Id: hal-01764191 \\ https://hal.inria.fr/hal-01764191}

Submitted on 11 Apr 2018

HAL is a multi-disciplinary open access archive for the deposit and dissemination of scientific research documents, whether they are published or not. The documents may come from teaching and research institutions in France or abroad, or from public or private research centers.
L'archive ouverte pluridisciplinaire HAL, est destinée au dépôt et à la diffusion de documents scientifiques de niveau recherche, publiés ou non, émanant des établissements d'enseignement et de recherche français ou étrangers, des laboratoires publics ou privés.

\section{(c)(1)}

Distributed under a Creative Commons Attribution| 4.0 International License 


\title{
BIM-FM and Information Requirements Management: Missing Links in the AEC and FM Interface
}

\author{
Julie Jupp ${ }^{1} \&$ Ramsey Awad ${ }^{2}$ \\ ${ }^{1}$ University of Technology Sydney, Australia \\ ${ }^{2}$ University of Newcastle, Australia \\ julie.jupp@uts.edu.au,ramsey.awad@newcastle.edu.au
}

\begin{abstract}
A steady shift in the value added from building information modelling (BIM) to architectural, engineering and construction (AEC) activities to those of facilities management (FM) is seeing increasing emphasis on whole-of-life thinking and associated information requirements management practices. Little is known about the process of identifying, documenting, generating and harmonizing BIM data inputs with FM data outputs in the Australian construction industry. Grounded on empirical evidence from a case study that transverses client and project team perspectives, this exploratory paper identifies missing links in the AEC and FM interface. The study describes the issues surrounding the collection and harmonization of BIM data inputs (as-built deliverables at handover) and the identification of (and connection to) FM data outputs. With the limitation of an exploratory and interpretive case study, the intention is to provide a contribution to academics and practitioners with grounded, stakeholder-related insights.
\end{abstract}

Keywords Building information modelling, Facilities Management, Information Requirements Management, Case study.

\section{Introduction}

Much has been written about the technologies, processes and policies underpinning the implementation of BIM in the AEC/FM industry. With regard to case studies on the implementation of BIM-FM fewer investigations exist. The technologies, processes, policies and people required to support the collection, harmonization and connection of BIM data inputs to FM data outputs are yet to be mapped relative to the boundary conditions of a client's operations and the new building project being procured. Existing research describes BIM-FM projects as an often ad-hoc discovery and learning process with a lack of a clear implementation 'methodology' defined at the outset of the project to support the specification and management of information requirements. Further, case studies on this topic, have in part, reflected large pilot projects undertaken by Tier 1 contractors in the delivery of public works projects. This perspective on 'best' practice can diminish industry to those leading enterprises that have achieved high levels of internal integration in their approach to digital engineering. A look behind the scenes reveals a not less powerful and absorbing ecosystem amongst smaller Tier 2/ 3 firms with more modest levels of maturity in internal integration and a distinct lack of BIM-FM know-how. A glimpse at the daily business of such project initiatives unveils a raft of interdependent issues confronting BIM for FM implementation. 
High levels of uncertainty compound the challenges surrounding the start of a BIM-FM initiative. AEC teams and owners inexperienced in the application of BIM technologies, processes and protocols for use in the operational phases increases the technical risks, which surround the scope and difficulty of the BIM-FM implementation problem not being well understood (or defined) at the outset. Owners should first undergo digital FM transformations internally so as to align with the newly available data and capabilities of BIM-enabled FM. Thus, in parallel to managing internal organizational change, owners requiring the delivery of 'FM-ready' BIM datasets by project teams prior to the completion of their own FM integration initiatives - and therefore without careful specification of the information requirements are managing complex problems with high levels of uncertainty. The owner's changing organizational context and any connected project environment will therefore be characterized by rapid technological change and high levels of learning. AEC/FM stakeholders may therefore be required to extend beyond their traditional roles, responsibilities and scope of works.

Recent works have studied BIM-FM implementation challenges (e.g., see [1-7]). Difficulties faced by Australian AEC/FM stakeholders in this context remain largely undocumented. A lack of understanding in the Australian AEC/FM industry therefore exists relative to $\mathrm{AEC}$ and $\mathrm{FM}$ information requirements specification and management process and as a consequence, AEC and owner/operator decision making are being based on 'acts-of-faith'. This research study is therefore an attempt unpack the complexity surrounding information requirements management and elucidate the nature and structure of undertaking a BIM for FM initiative. Grounded on empirical evidence from a case study on a university building project with an inexperienced client and a Tier 2 contractor, we explore the BIM-FM implementation problem using the following two-part research question:

[RQ1:] How are BIM-FM information requirements specified and managed across project phases - relative to the identification, documentation, collection and harmonization of data inputs; and connection with FM data outputs? And

[RQ2] What links are missing in the AEC and FM stakeholders interface when specifying and managing BIM for FM information requirements?

The remainder of the paper is divided into four sections. Section 2 provides a brief review of related literature. Section 3 presents the research methodology, data collection and analysis methods. Section 4 presents the findings and discusses the issues identified. Section 5 concludes the paper, synthesizing the observations in a discussion before identifying the limitations and implications of the research.

\section{Background}

ISO15686-1 defines maintenance as the "Combination of all technical and associated administrative actions during service life to retain a building or its parts in a state in which it can perform its required functions." $[8, \mathrm{p} 2]$. In the standardization initiatives surrounding the application of BIM to the maintenance phase of a facility, a central issue has been how to specify, document, generate, collect, structure and exchange 
digital information to enable its connection across different disciplines participating throughout the building life cycle. Recent studies focusing on the value of as-built datasets (virtual building models) to operations and facilities maintenance (O\&FM), highlight difficulties surrounding their creation $[9,10]$, demonstrating that there is often no clear strategy for data harmonization or keeping the BIM model "alive" after handover. Whilst proponents of BIM assert its effectiveness in enabling process integration and information management (avoiding information losses incurred when transitioning from one team to another), a critical assumption remains that each group has the ability to "add to and reference back to all information they acquire during their period of contribution to the BIM model" [11]. Such aspirations for fully coordinated datasets and seamless information management appear to be far from reality.

The building life cycle is both complex and fragmented [12], and across each phase, information requirements differ and serve different purposes [13]. Whilst, information generated upstream should be available in downstream phases in a continuous data flow [14], research shows that current industry practices result in disconnected islands of data at each phase [1-5]; when data is communicated, the transfer most often occurs as a one-off event to the subsequent phase [13]. Consequently, the harmonization and connection of information to O\&FM remains limited. As O\&FM activities are multidisciplinary, there are extensive information requirements. The flow of information is restricted largely due to ineffective and inefficient exchange of information among AEC/FM stakeholders and each discipline's unique information systems. Building design and production call for information to be exchanged between AEC stakeholders across project phases to coordinate and integrate the various building systems within the project. Building O\&FM remains largely disconnected from project phases with handover and commissioning activities being poorly defined, coordinated and managed due to schedule and budget constraints [15]. The O\&FM data 'island' therefore suffers from ineffective and inefficient use as it requires the delivery of accurate and high quality data at building handover, which largely depends on having construction, supplier and O\&FM expertise involved earlier in the design phases.

To maximize the use of buildings and minimize operational costs, the requirements of O\&FM must be considered from project inception and verified throughout each phase [16]. Various initiatives have tried to support this aim. For example, the buildingSMART Data Dictionary (bSDD) [17], known as the International Framework for Dictionaries (IFD) and defined in ISO 12006-3 [18], was developed as a terminology standard for BIM libraries and ontologies. The IFD library is most useful when used in combination with the Industry Foundation Class (IFC) schema and the Information Delivery Manual (IDM) approach. Together, this approach is capable of describing what kind of information is exchanged by providing a mechanism that allows the creation of unique IFD IDs, connecting information from existing databases to IFC (Industry Foundation Classes) data models [19]. IFC4 is the latest version of the ISO 16739 standard [20] and has brought significantly improvements in energy and performance analysis, environmental impact values, integration of ifcXML and mvdXML into specification and improved documentation [21]. 
A further initiative to span the AEC-FM divide is the COBie (Construction Operations Building information exchange) specification [22], which denotes how information may be captured during design and construction and provided to facility operators. This method provides a spreadsheet of the same exact information available in the IFC file in a way that is easier to human to understand. The main objective of COBie is to provide for: (i) an IFC reference standard so as to directly support software information exchange, and (ii) a spreadsheet that can be used to capture building and operations data. Supporting the use of COBie is the Facility Management Handover Model View Definition [22]. The model view definition (MVD) identifies all the objects in IFC file necessary to support COBie and the relationship between these objects. MVD defines the process for determining the appropriate exchange requirements from the IFC schema for any particular use, representing them in an Information Delivery Manual (IDM) [23] However, there are few studies documenting the implementation of this integrated approach to the capture of processes and exchange requirements.

The application of OmniClass has also progressed the pursuit of BIM-FM. OmniClass is a standard that combines multiple existing classification systems for many subjects into a single unifying system, which is based on ISO 12006-2, Organization of Information about Constructions Works - Part 2: Framework for Classification of Information [24]. However, whilst this standard establishes common concepts used in building information exchange, it is a guidance document and has no explicit rules for implementation [25]. As a result, Omniclass can be implemented in different ways and, used on its own, is seen by some as having the potential to create inconsistencies [13] and should be used in conjunction with e.g., IFD, so as to enable the access and viewing of data via an unambiguous classification system.

In the United Kingdom (UK), the HM Government's BIM Programme has mandated 'Level 2 BIM' (file-based collaboration and library management) on all centrallyprocured Government projects, which was enforced mid-2016. Within this policy, the need for the provision of structured data for asset information models is recognised in the UK PAS1192-3:2014, 'Specification for information management for the operational phase of assets using building information modelling' [26]. PAS11923:2014 specifies an information management methodology for the operational phase of assets based on open BIM standards and data specifications - COBie [26]. PAS 11923:2014 utilizes a hierarchical structure encompassing four information requirements specification processes, namely the: organization information requirements (OIR), employer information requirements (EIR), asset information requirements (AIR) and project information model (PIM). The support of asset and facility managers in specifying their information needs takes the form of an Asset Information Model (AIM) and is thus aimed at enabling the production of a data model that can be linked to the as-built BIM model, which contains all digital information required for operation [26]. It is intended that the AIM then be communicated to lead designers and contractors. However to meet the information needs of owner/operators, accurate data must be generated, collected and exchanged throughout project delivery phases, and not left as a 'clean-up' activity that occurs just prior to- or after- building handover. From this perspective, the process of defining EIR by the owner - and upon which the AIM depends - is fraught with difficulty [13]. Missing links in the AEC-FM interface arise 
from a lack of quality control mechanisms, since whilst the minimum set of information requirements for an EIR is specified in the PAS 1192-2 [27], an EIR's definition necessitates requirements for all disciplines involved in project delivery and operations.

Further, EIR specifications do not currently account for a method to define, collect and document data input requirements from AEC and FM stakeholders. This deficiency reflects other critiques of BIM for FM initiatives to date, which demonstrate implementation deficiencies relative to the early involvement of O\&FM expertise, i.e., the 'closed-loop' engineering problem, [28, 29]. This is complicated by a lack of detailed quality control systems in construction that facilitate digital deliverables during project phases, and whilst the definition of data drops with specifications for information content and granularity, (e.g., COBie data drops in the UK [16]), can help capture and verify client requirements are being met, the specification of progressive model content schemas which rely of definitions of the 'Level of Development' (LOD), are problematic. Bolpagni [31] highlights the difficulties arising from a lack of a "univocal approach to the definition and management of the content of a virtual building model". Several definitions of LOD exist, (e.g., 'Level of Detail' and Level of Information), resulting in subjectivity, interpretation and inconsistency.

Implementing BIM-FM not only requires the delivery of accurate 'as-built' datasets at project completion that fulfill a client's (often undefined) request for an 'FM-ready' BIM model, but also requires that operations and maintenance (O\&M) documentation, asset hierarchies, and building classification systems correspond with the metadata contained in the model. These responsibilities often fall on (inexperienced) main contractors to audit, verify and harmonize data inputs with the owner's O\&FM data outputs. In doing so, it is necessary to consider the owner's existing and future information systems, processes and protocols, whilst at the same time working with design consultants and subcontractors to ensure accurate, high quality data inputs. Where a lack of integration across an owner's existing O\&FM systems and inconsistencies in operational workflows exist, the task of data harmonization can become an intractable one. An integrated and consistent approach to digital FM is therefore primary in obtaining the benefits of BIM.

Within this context, regulatory frameworks and in particular contract procurement methods will have a major impact on the successful delivery of as-built datasets and of the timely harmonization of data inputs with O\&FM data outputs. The nature of most contractual frameworks applied to govern the design, construction and commissioning phases of projects predate the use of BIM [32] - particularly the delivery of digital assets for the purpose of building life-cycle management. Contractual frameworks can therefore obstruct than rather support the use of BIM in O\&FM. Compounding these challenges are the constraints of low levels of client-side knowledge and modest BIM and digital FM implementation budgets. Studies that describe BIM for FM typically focus on the project delivery phases and overlook client-side challenges surrounding the continuous improvement and ongoing investment requirements that BIM-FM approaches necessitate in operations. In a study on the Sydney Opera House (SOH), the nature and substantial size of ongoing investment in a BIM-FM strategy is highlighted [33]. A substantial investment in initial modelling costs, continuing costs of 
maintaining and managing models, developing flexible and scalable database integration platforms combined with the development costs of a visual engine (or BIMFM interface) present a variety of known unknowns as well as unknown unknowns that must be risk managed from the outset.

\section{Research Methodology}

The objective of this research is to investigate challenges surrounding the specification and management of information requirements that support the use of BIM in FM, with focus on the AEC and FM interface. We selected an exploratory research strategy, based on a single-case study approach, which used an interpretive epistemological perspective [34]. From November 2016 to March 2017 an intensive study into a Tier 2 construction firm ('ContractorT2') and a university client and O\&FM team ('UniClient') was undertaken. The study concentrated on the interfaces between stakeholders during the design and construction of a new building project. As case study research strongly relies on the case context [34], characteristics of ContractorT2, UniClient and project background are outlined below.

ContractorT2 is an Australian owned private company that derives the majority of its revenue from the provision of construction services. From a financial viewpoint, ContractorT2 features revenues of approximately AUD \$520 million and comprises approximately 400 employees. As a Tier 2 contractor, the company aims to differentiate its construction services via innovation and quality from competitors. For this purpose, ContractorT2 approaches projects based on an integrated and accredited project management system with services are aimed at providing value and risk management to their clients using BIM to ensure a best value for money with a smooth delivery.

UniClient is an Australian public university with approximately 40,000 students. The building project that forms the setting of this case study is a 16 story building and is the latest and most significant development in the university's campus master plan. Running concurrently with the design and construction of the building project, UniClient is defining a new "concept of building operation" to determine seven key aspects of asset management: (a) high level operating approach, (b) required roles and resources, (c) scope of assets to be defined in asset management, (d) required functions and processes across campus, (e) interfaces to financial management and other university systems, (f) mapping of workflows, and (g) reporting and auditing systems. The approach of UniClient's digital FM transformation strategy is to ensure it can be used not just for the new building, but existing buildings as the transformation process will be a multi-year, multi-project program of projects. Further, key change management activities are underway to support UniClients digital transformation moving from a "reactive repair and replace" culture to a "proactive and predictive" one.

UniClient's technology brief informed ContractorT2 (and other project stakeholders) via its translation into the project's BIM Execution Plan (BEP). The client's aspirations centered on BIM's potential to deliver a fully documented building at handover with supporting O\&M documentation that can be used and updated throughout the 
operations phase. To deliver data inputs to UniClient's O\&FM systems, a range of FM functions and systems were accounted for. The main requirements were:

- BIM-based input data to ensure a record of initial asset information, and as a means of documenting change to the campus' built environment.

- BIM-based input data to automatically link and download to existing computer-aided facility management (CAFM) asset register and management module. Data relating to the asset should be posited in the CAFM and linked back into the BIM model.

- Use of Construction Operations Building information exchange (COBie) protocol to enable asset data synchronisation between model and CAFM asset mgmt. module.

- Interface between CAFM, building management system (BMS) and Security Systems to allow for live data feed, changing from a reactive to pro-active fault tolerant culture.

- Integration with Geographic Information System (GIS) data to map in ground services and make information available to CAFM.

- Interface with space management system using web-based interface for space planning, operations and reporting activities.

Semi-structured interviews built the main foundations of the study, with other sources of evidence informing the findings, including archival records (e.g., strategic documents and management plans) and digital artifacts (e.g., software applications and models). Overall, 12 interviews across the two organizations were undertaken on a faceto-face and remote basis. In line with the scope of the BIM-FM implementation problem, we included stakeholders from across the design consultant and subcontracting teams. Following the exploratory nature of the research, we adapted grounded theory techniques [35] for data analysis. Open, axial, and selective coding procedures were then utilized. During the coding procedures, NVIVO 10 was utilized to assure consistent and efficient data analysis.

\section{Findings}

In the case study, missing links in the AEC-FM interface of BIM-FM implementation were identified. Table 1 presents the gaps and obstacles perceived by interviewees in order of the greatest coding frequency, with each of the ten issues discussed below.

Table 1. Gaps and obstackles to the specification and management of information requirements

\begin{tabular}{l|l}
\hline No. & Challenge \\
\hline$\# 1$ & Lack of Application \& Awareness of Intl. Standards \& Guidelines \\
\hline$\# 2$ & Deficiencies in Contractual Frameworks and BIM Execution Plans \\
\hline$\# 3$ & Missing Stakeholders during Design Phases \\
\hline$\# 4$ & Lack of IFC and COBie Support for Asset Data \\
\hline$\# 5$ & Multiple Breaks in Information Flow \\
\hline$\# 6$ & Heterogeneous Data Inputs and Outputs \\
\hline$\# 7$ & Deficiencies in \& Varying Commitment to Modelling \\
\hline$\# 8$ & Lack of Whole-of-life Thinking \\
\hline$\# 9$ & Lack of BIM-FM competency assessment \\
\hline$\# 10$ & Deficiencies in BIM-FM Benefits and Business Cases \\
\hline
\end{tabular}

\#1 - Lack of Application \& Awareness of Intl. Standards \& Guidelines: AEC stakeholders had limited detailed knowledge about the application of international standards and guides (e.g., those established in the UK and US) to support information 
requirements specification and quality control management processes. The AEC project team lacked information about the assets and actual operations, and did not have a guide to support the collection of requirements. The information requirements specification and quality management methods utilized lacked in appropriate policy coverage across project delivery. It was perceived that the focus of existing approaches to BIM lies on the design and production phases, with processes and protocols to support handover, commissioning and operations requirements largely being neglected. Further, feedback loops after practical completion had not been planned.

\#2 - Deficiencies in Contractual Frameworks and BIM Execution Plans (BEP): The BEP was perceived as a multi-layered and extensive document addressing design consultants, contractor and suppliers. However, being a construct-only contract, ContractorT2 was not able to control of the entire collaborative effort (as in a DnC or Managing Contractor delivery), and therefore the BEP was not able to consider information requirements across the entire project in a thorough and timely manner. Modelling requirements and workflows were therefore ill-defined in light of the model uses that were required, particularly for the ultimate purpose of supporting O\&FM.

\#3 - Missing Stakeholders during Early Design Phases: During the pre-construction phase, the contractor's information management task was complicated by their limited ability to affect the requirements management process, and in particular data drops. This was largely seen to be due to the absence of suppliers. Without appropriate contractual agreements and protocols in place, collaboration was an obstacle to key data-drops and milestones during design. Despite client-side BIM-FM objectives, AEC and FM stakeholders remained disconnected. Further, whilst information exchange was given a high priority, due to challenges rooted in different information systems, this issue was left largely unresolved. The software itself was not seen as the bottleneck, but rather the lack of early stakeholder engagement and information sharing.

\#4 - Lack of IFC and COBie Support for Asset Data: The use of IFC4 and COBie were initially tested by the contractor so as to map asset register requirements in IFC4 and COBie 2.4. Testing on the project (and previous projects) by the contractor had shown that some of the main requirements were not directly supported. Whilst some of the non-supported fields were part of data drops, gaps were identified in O\&FM information (e.g., costs breakdown, asset value, accumulated depreciation, and sources of spare parts). Whilst the possibility of including additional information in IFC and COBie files using custom property sets was raised, consensus within ContractorT2 was that this process would be too time consuming and relied on the design team and subcontractor's cooperation as it was necessary to first identify which components were not correctly exported to IFC, before then defining correct IFC types and entities.

\#5 - Multiple Breaks in Information Flow: As a result of the above issue coupled with the discipline-specific information system landscapes, the contractor was confronted with multiple occurrences of breaks in information flow across project phases. A seamless transition from requirements specification to design and engineering, simulation and analysis, to construction planning, manufacturing, and onsite construction did not exist. Building model data exported from ArchiCAD and imported into Revit interrupted information flows and reduced AEC stakeholder confidence in model consistency, reinforcing information 'silos'. 
\#6 - Heterogeneous Data Inputs and Outputs: From a tool perspective, AEC and FM stakeholders were challenged by boundary conditions such as information protocols and software standards. On the one hand, in working with multiple consultants and subcontractors it was difficult for the main contractor to impose different data input requirements, yet in some cases failure to do so resulted in redundant design consultant models. On the other hand, the owner had difficulty in specifying data output requirements and mapping functions from the various asset and FM software used. Further, the CAD tools used across project stakeholders reflected their own tool portfolio. Heterogeneous and contrarious information requirements resulted and management of data inputs by contractor was significantly challenged.

\#7 - Deficiencies in \& Varying Commitment to Modelling: Deficient use of modeling standards and varying levels of modelling commitment across both AEC and FM teams was a common theme. Whilst for many interviewees, BIM-FM was typically equated with a central repository for building data, the significance of human factors was emphasized relative to inconsistencies across information standards, software and modelling practices inherent within that repository. Understanding the relevance of quality control systems and information requirements management as a holistic strategy entrenched into the project culture was not an established thinking.

\#8 - Lack of Whole-of-life Thinking: A lack of a 'lifecycle' approach in the development and use of BIM-FM protocols presented a significant challenge. Without whole-of-life thinking being driven from the client, the O\&FM needs could not be addressed adequately by the contractor. Although the client had a vested interest in the long-terms benefits of BIM, the BEP and contractual framework did not include key methods to attain these goals, (e.g., PAS 1192-3:2014 and EIR for Facilities and Asset Management).

\#9 - Lack of BIM-FM competency assessment: There was a consensus that key members of the project stakeholders did not yet have the required level of BIM-FM knowledge or experience required. This meant that the competencies required to deliver successful outcomes were either being learnt on-the-fly or resulting in poor data delivery. The lack of BIM-FM competency assessment and the willingness of the client to blindly trust the project teams presents a substantial risk to the realization of BIMFM's return on investment. However whilst competency assessment was seen as important it was not perceived as being an essential part of tender processes. It was however seen as critical to distinguish between those BIM-FM competencies that are essential prerequisites and those that could be learned on the job.

\#10 - Deficiencies in BIM-FM Benefits and Business Case: Whilst benefits for adopting BIM in the AEC sector are well known, the client organization was in the early stages of developing and implementing a business case for BIM-FM campus wide. The common perception was that most of the documented, quantifiable benefits of BIM are related to the design and production phases, with a lack of evidence and metrics on the benefits BIM to FM. The case to support the required (and ongoing) investment in BIMFM was seen by the client to rely on its value to not only supporting FM functionalities - such as space and asset management - but also in managing operational risks; e.g., code compliance, asset certification, environmental performance monitoring, emergency response, and predictive operational safety and scenario planning. Whilst 
the existence of an effective benefits delivery and management process was lacking within the client organization it was perceived as necessary to supporting mutual cooperation of project and operational management functions.

\section{Discussion}

Some of the identified challenges are similar to related BIM-FM studies, e.g., [1-7], whereas others identify issues specific to this study and its context. For example, challenges \#2 ("Deficiencies in Contractual Frameworks and BEP") and \#4 ("Lack of IFC and COBie Support for Asset Register Data") are counted among these. Wider influences are identified relative to how Australian AEC and FM stakeholders are impacted by and can influence each issue. To illustrate this, Table 2 provides a stakeholder analysis of 'impacted by' and 'power to influence' the ten challenges identified relative to the project context, shown as high $(h)$, medium $(m)$, or low $(l)$.

Table 2. Stakeholder analysis of information requirements management issues

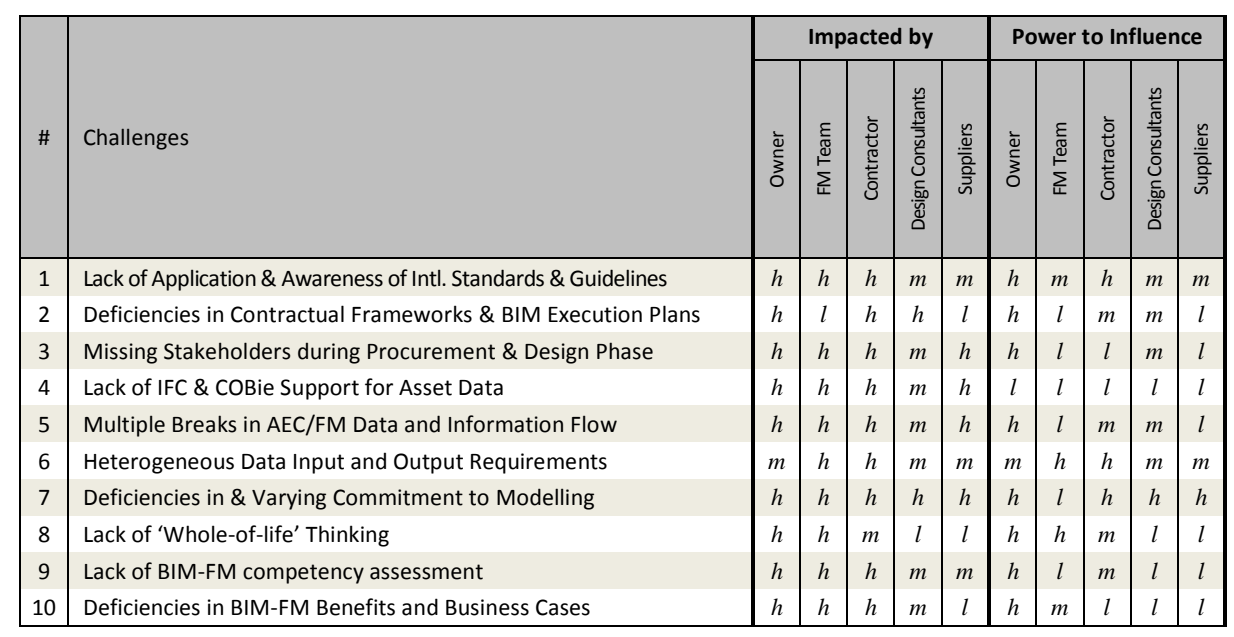

From the analysis, it can be observed that not only the owner and contractor are impacted by the issues identified but also the FM team, design consultants, suppliers. Few challenges therefore exist that individual stakeholders can address alone. Referring back to the underlying research questions, the findings reinforce that the specification and management of information requirements are constrained by conditions that span project phases and discipline responsibilities but cannot be influenced by all equally.

These ten issues identified contribute to the research discussion in a number of ways. First, these challenges relate to the specification and management of information requirements across project phases and discipline responsibilities, which highlights an underlying deficiency surrounding the lack of quality control mechanisms in information requirements management processes in construction. Quality management systems (QMS) established in conformity with ISO 9000 standards have a twenty-year tradition in the automotive and aerospace industries. The most recent issue of the ISO 
9001 standard (2015) focuses on technology risk management, the innovation implementation process, and information management. Secondly, with respect to the loosely coupled stakeholders in the BIM-FM landscape, although the development of a QMS for information management will require the joint optimization of related processes and workflows, development will be difficult. As many technological, processual, and policy steps have to be climbed on the path to successful BIM-FM implementation, transformation of existing approaches to quality control in construction will be gradual. Finally, whilst this is a starting point, integrated procurement, organizational change management and detailed commissioning processes represent other essential activities. The relevance of the social component of information requirements management in the BIM-FM implementation process is highlighted by the study's findings; as a dynamic socio-technical system, developing QMS for BIM-FM requires further research in this regard.

The advantages of a single-case study go hand-in-hand with its limitations. Although a typical construction company and client was investigated, research findings are not representative or generalizable across the construction industry. Data analysis is also interpretive and the exploratory nature of the study does not ensure exhaustiveness. However the study raises potential directions for further research. Validating the challenges identified using a mixed method or quantitative research design would provide further insight. To capture a broader perspective, multiple case studies may be useful to investigate other project contexts and provide a valuable corpus to begin a comparison. From this perspective, it may then be possible to map identified challenges with potential solutions. For these activities this study acts as a point of origin.

\section{References}

1. Whyte, J., Lindkvist, C. \& Ibrahim N.H., (2011). From Projects into Operations: Lessons for Data Handover. Uni. of Reading, DIRC. Working Paper. www.reading.ac.uk/

2. Arayici, Y., Onyenobi T. \& Egbu, C., (2012). Building information modelling (BIM) for facilities management (FM): The MediaCity case study approach. International Journal of 3-D Information Modeling (IJ3DIM) 1(1): 55-73.

3. Kelly, G., Serginson, M., Lockley, S., Dawood, N., \& Kassem, M. (2013). BIM for facility management: a review and a case study investigating the value and challenges. In Proc. of the 13th Intl. Conf. on Construction Applications of Virtual Reality (pp. 30-31).

4. Codinhoto, R., \& Kiviniemi, A. (2014). BIM-FM: A Case Support for Business Life Cycle. In IFIP Intl. Conf. on PLM (pp. 63-74). Springer Berlin Heidelberg.

5. Kassem, M., Kelly, G., Dawood, N., Serginson, M., \& Lockley, S. (2015). BIM in facilities management applications: a case study of a large university complex. Built Environment Project and Asset Management, 5(3), 261-277.

6. Ibrahim, K. F., Abanda, F. H., Vidalakis, C., \& Woods, G. (2016). BIM-FM: Input versus Output data, Proc. of the 33rd CIB W78 Conference, Oct. 31 st - Nov. 2nd, Bris., Australia.

7. Ashworth, S., Tucker, M., Druhmann, C., \& Kassem, M. (2016). Integration of FM expertise and end user needs in the BIM process using the Employer's Information Requirements (EIR). In Proceedings of CIB World Building Congress (Vol. 5).

8. ISO (2011). ISO 15686-1, Buildings and constructed assets: Service life planning - Part 1: General principles and framework, Intl. Org. for Standardization, Geneva, Switzerland.

9. Ashworth, S., Tucker, M., \& Druhmann, C. (2016). The Role of FM in Preparing a BIM Strategy and Employer's Information Requirements (EIR) to Align with Client Asset Management Strategy. Published 2016, 218.

10. Xuesong, L., Eybpoosh M. \& Akinci B. (2012). Developing As-built Building Information Model Using Construction Process History Captured by a Laser Scanner and a Camera. Construction Research Congress. 
11. Bew, M., \& Underwood, J. (2010). Delivering BIM to the UK Market. In J. Underwood \& I. Umit (Eds.), Handbook of Research on Building Information Modeling and Construction Informatics: Concepts and Technologies (pp. 30-64): IGI Global.

12. Hitchcock, R. J. (1995). Improving building life-cycle information management through documentation and communication of project objectives. Proceedings of Modeling of Buildings through their Life-Cycle, 150, 358.

13. Pinheiro, S. V., Corry, E., \& O'Donnell, J. T. (2015). Requirements for a BIM-Based LifeCycle Performance Evaluation Framework to Enable Optimum Building Operation. In 32nd Intl. CIB W78 Conf., Eindhoven, The Netherlands, 27-29 Oct. 2015 (pp. 639-648). EUT.

14. Man, Q., Wang, Y., Li, H., Chang, Y., \& Martin, S. (2013). Life-cycle information flow management system. Intl. J. of Digital Content Technology and its Applications, 7(8), 857.

15. Carmichael, D. G. (2006). Project planning, and control. Routledge.

16. BSRIA (2009), The Soft Landings Framework, BSRIA.

17. ISO Standard (2007). ISO 12006-3:2007 Building construction: Organization of information about construction works, Part 3: Framework for object-oriented information. 2007.

18. buildingSMART (2012) IFD Library for buildingSMART 2012.

19. Laakso, M. \& Kiviniemi, A., (2012). The IFC standard - A review of history, development, and standardization. Jrl. of Info. Tech. in Constr. (ITcon), 17, pp.134-161.

20. ISO Standard (2013). ISO 16739:2013 Industry Foundation Classes (IFC) for data sharing in the construction and facility management industries, 2013.

21. Liebich, T., (2013). IFC4 - The New buildingSMART Standard: What's new in IFC4?

22. East, W.E (2007). Construction Operation Building Information Exchange. USACE ERDC.

23. Belsky, M., Sacks, R. A \& Brilakis, I., (2016) Semantic Enrichment Engine for Building Information Modelling. Computer-Aided Civil and Infrastructure Engineering, 261-274.

24. ISO 12006-2 (2015). Building construction -- Organization of information about construction works - Part 2: Framework for classification. ISO.

25. Cemesova, A. (2013). Enhancing BIM-based data transfer to support the design of low energy buildings (Doctoral dissertation, Cardiff University).

26. BSi, (2014). PAS 1192-3:2014 Specification for information management for the operational phase of assets using BIM, London: BSi Limited.

27. BSi (2013). PAS 1192-2:2013 - Specification for information management of the capital delivery phase of construction projects using building information modelling, British Standards Institution, London: BSI.

28. Anderson, A., Marsters, A., Sturts Dossick, C., \& Neff, G. (2012). Construction to Operations Exchange: Challenges of Implementation COBie and BIM in a Large Owner Organization. Construction Research Congress, Purdue University, IN, May, 15pp.

29. Reefman, R. \& van Nederveen, S. (2012). A Controlled Integral Product Model (IPM $\left.{ }^{\circledR}\right)$ in Building and Construction, Proceedings of CIB W78-W102 2011: Intl Conf., France.

30. Jupp, J. R. (2013). Incomplete BIM implementation: Exploring challenges and the role of PLM functions. In IFIP Intl. Conf. on PLMt (pp. 630-640). Springer Berlin Heidelberg.

31. Bolpagni, M., \& Ciribini, A. L. C. Bolpagni, M., \& Ciribini, A.L.C. (2016). The Information Modeling and the Progression of Data-Driven Projects. Proceedings of WBC16, Finland.

32. London, K., Singh, V., Taylor, C., Gu, N. \& Brankovic, L. (2008). Building information modelling project decision support framework. In: Dainty, A (Ed.), Proc. 24th Annual ARCOM Conference, Cardiff, UK. Assoc. of Researchers in Const. Mgmt, Vol. 2, 655-64.

33. Ebbesen, P., Karlshøj, J., Bonke, S., \& Jensen, P. A. (2016). Information System Strategies in Facilities Management-Based on Five Process Studies. In Pro. of Cfm's 2nd Nordic Conf.: FM Research and Practice: Does FM Contribute To Happiness in the Nordic Countries.

34. Yin, R.K. (2009) Case Study Research: Design and Methods, 4th Ed, SAGE Pub. CA.

35. Strauss, A.L., \& Corbin, J.M. (1997). Grounded Theory in Practice. Thousand Oaks: Sage. 